\title{
The bandwidth reduction effect of photoluminescence spectrum from nanostructures on silicon
}

\author{
Han-Wei Wu, Shu-Jia Shiu, Chao-Jei Huang, Chia-Wei Tsai, and Ching-Fuh Lin ${ }^{+}$ \\ Graduate institute of Electro-Optical Engineering, National Taiwan University, \\ No.1, Sec. 4, Roosevelt Road, Taipei, 106, Taiwan, R.O.C \\ ${ }^{+}$also with Graduate institute of Electronics Engineering and Dept. of Electrical Engineering
}

\begin{abstract}
We created nanostructures on bulk silicon and measured their photoluminescence (PL) spectra. The PL spectral width of the samples with nanostructures is much narrower than that of bulk silicon due to confinement of exciton.
\end{abstract}

During the research of silicon luminescence, we found that some nanostructures on silicon contributed to the reduction of the bandwidth of its photoluminescence (PL) spectrum under low temperature. The effects of the nanostructures are suspected to increase the relaxation time of excitons and to lower the energy of continuum excitons. Besides, excitons are one kind of bosons and undergo the Bose-Einstein distribution. Thus under low temperature and the quantum confinement effects [1] of the nanostructures, mass excitons will concentrate at the lowest energy level, similar to Bose-Einstein Condensation. We developed a new method to create pillar-like structure on silicon surface in nanometer scale. Then we measured the PL spectrum of our nanopillar structure on silicon under varied temperatures and pump powers. The pumping sources are $532 \mathrm{~nm}$ laser and $980 \mathrm{~nm}$ laser. Our samples were put in a chamber in which temperature can be controlled to $10 \mathrm{~K}$. After precise measurement and analysis, we found that at $10 \mathrm{~K}$ and pump power $50 \mathrm{~mW}$, the spectral width of PL spectrum of our nanopillar structure on silicon is reduced to $3.9 \mathrm{~nm}$, which is a very narrow linewidth. If we lower the pump power, the linewidth is even narrower. We established a theoretical model based on semiconductor physics and quantum mechanics to simulate the light emitting process of silicon. Then we use this model for curve fittings. After curve fitting and some analysis, the effects of our nanopillar structures under low temperature are found to reduce the linewidth of PL spectrum from about $10 \mathrm{~nm}$ to $3.9 \mathrm{~nm}$ (under same pump power) because the relaxation time is raised from about $200 \mathrm{fsec}$ to about $900 \mathrm{fsec}$ and the energy of continuum excitons is lowered. Several nanostructures on silicon have been experimented to have effects on the linewidth of the PL spectrum. We spun several sizes of $\mathrm{SiO} 2$ nanoparticles on silicon wafer, and measured the PL spectrum under $10 \mathrm{~K}$. The results showed that the sample with smaller $\mathrm{SiO} 2$ nanoparticles spun upon has narrower spectral linewidth (below $4 \mathrm{~nm}$ in wavelength under low pump power), and the intensity was higher (approximately $5 \sim 6$ times to that without spun-on $\mathrm{SiO} 2$ nanoparticles). This means that the photon energy is concentrated in the narrower spectral region.[2] We presume that the surface with $\mathrm{SiO} 2$ nanoparticles spun upon produces random potential traps and these traps would lower the kinetic energy of excitons created by laser illumination. The smallest particles (diameter $=10 \mathrm{~nm}$ ) cause most efficient confinement effect to excitons and so lead to the narrowest linewidth.

We had also spun silicon and $\mathrm{SiO} 2$ mixed nanoparticles on silicon and then heated the sample to let the Si particles on silicon wafer recrystalled. The PL spectral width of this structure is below $3 \mathrm{~nm}$ at pump power $50 \mathrm{~mW}$. If we lower the pump power, the spectral width could be lowered to about $2 \mathrm{~nm}$. This is the narrowest bandwidth of silicon observed in our knowledge. Narrower spectral width should be possible with optimized nanostructures. In the future, we expect that the nanostructures in Si could lead to BEC phenomenon of exciton in silicon.

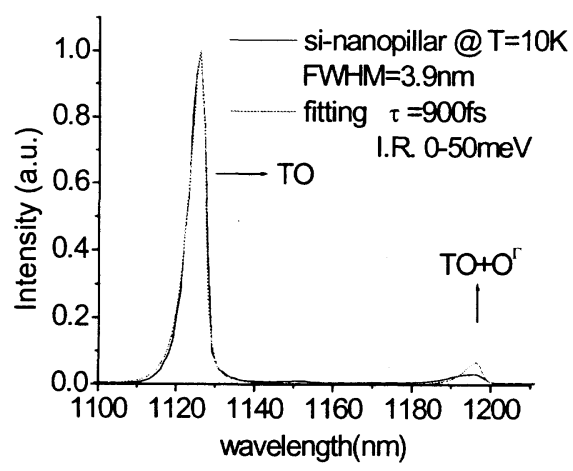

Fig. 1 PL spectrum of nanopillar structure at $10 \mathrm{~K}$.

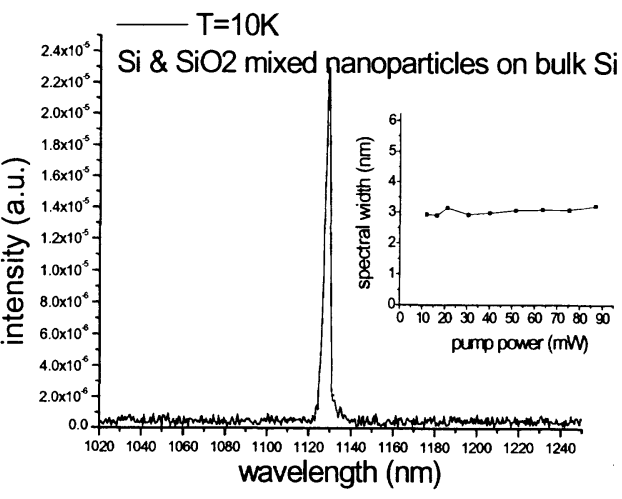

Fig.2 PL spectrum of new nanostructure at $10 \mathrm{~K}$.

References

[1] L.V. Butov, "Exciton condensation in coupled quantum wells", Solid State Communications 127 (2003) 89-98

[2]Paul Wright, "Quantum confinement effects in semiconductor clusters", Computational Part III Project(Churchill College, Cambridge 2000) 